\title{
Colloidal Drug Delivery Systems - Recent Advances With Polymeric Micelles ${ }^{\S}$
}

\author{
Karine Mondon, Robert Gurny, and Michael Möller*
}

\begin{abstract}
With the emergence of nanotechnology in drug delivery, colloidal systems and particularly polymeric micelles have attracted great attention. Polymeric micelles formed by the self-assembly of amphiphilic copolymers adopt a core-shell structure, which can be loaded with drugs and used as drug delivery systems for various medical applications. The most interesting aspects involve extended blood circulation times and stability upon dilution, which enable polymeric micelles to accumulate in tumor or inflammation sites due to the enhanced permeation and retention effect (EPR). In the first part of this paper polymeric micelles with different morphologies and different circulating-, active- and passive targeting, and stimuli responsive properties will be reviewed. Furthermore amphiphilic block copolymers of different compositions for pharmaceutical micelle formulations will be discussed. The hydrophilic block is often composed of the biocompatible polyethylene glycol (PEG), whereas diverse polymers are used for the hydrophobic block. The biodegradable and biocompatible polylactide (PLA) is one of the most tested core-forming blocks for micelles, in part because of approval by the FDA for numerous drug products for use in humans. However, PLA has limitations with respect to the incorporation of poorly water-soluble drugs. Considering this we will present in the second part of this paper briefly our strategy and contribution to overcome these limitations and recent results for the envisioned application of these micelles in the field of cancer treatment. In order to increase the hydrophobicity of PLA methyl groups are substituted by more hydrophobic hexyl groups. The hexyl-substituted polylactides in combination with PEG form the amphiphilic block copolymers PEG-hexPLA, which self-assemble in aqueous solution into stable spherical, homogenous micelles with a diameter of 20-45 nm. The increased hydrophobicity of the hexPLA micelle core leads to higher incorporation rates of hydrophobic drugs, like the poorly water soluble photosensitizer, meso-tetra(p-hydroxyphenyl)porphine (THPP). THPP water solubility is increased 200 -fold using these micelles. Their application in photodynamic therapy (PDT), coupled with the increased accumulation of the photosensitizer in a tumor, followed by irradiation at a special wavelength, should lead to tumor death. The encouraging drug incorporation results reveal the potential of hexyl-substituted polylactide micelles as drug carriers for PDT applications.
\end{abstract}

Keywords: Biodegradable polymers · Block copolymers · Drug delivery · Polymeric micelles . Substituted polylactides

${ }^{\star}$ Correspondence: Dr. M. Möller

School of Pharmaceutical Sciences

University of Geneva, University of Lausanne

Department of Pharmaceutics and Biopharmaceutics

30, Quai Ernest Ansermet

$\mathrm{CH}-1211$ Genève 4

Tel.: +41223793132

Fax: +4122 3796567

E-mail: Michael.Moeller@pharm.unige.ch

$\S$ This paper was presented in part at the 21st Confe-

rence of the European Colloid and Interface Society

'ECIS 2007', September 10-14, 2007, in Geneva

\section{Overview of Polymeric Micelles as Drug Delivery Systems}

In the first part of this article we will give a brief overview on polymeric micelles as drug delivery systems. Specific properties of polymeric micelles will be outlined with an emphasis on the different compositions of widely used amphiphilic copolymers. In a second part, recent developments from our own research on hydrophobic substituted PLA-based copolymers and their possible use as micellar carriers of poorly water soluble drugs for cancer treatment will be presented.

One very important aspect of pharmaceutical research is the development of novel drug carriers, which can deliver drugs to the right pathological site, at the right time, in the right dose, without affecting surrounding healthy tissues. In order to achieve this, nanotechnology has been pushed into the spotlight with the exploration of various colloidal drug delivery systems like nanospheres, nanocapsules, micelles, liposomes, nanoemulsions, nanogels, lipid base nanoparticles, dendrimer nanocomposites, and others. ${ }^{[1]}$ Among these systems, micelles have gained increased interest in the last 15 years. The number of patents and publications is increasing exponentially. The number of published papers in 1990 dealing with 'polymeric micelles' was about one hundred, whereas in 2007 it was eight times higher (Fig. 1).[2] Numerous excellent reviews are available in the literature describing micellar carrier systems for contrast agents in the imaging and diagnostic field, ${ }^{[3]}$ as drug carriers ${ }^{[4,5]}$ for cancer, ${ }^{[6-8]}$ and for gene and photodynamic therapy. ${ }^{[9]}$

Polymeric micelles for pharmaceutical applications can be formed from amphiphilic copolymers with different architectures (Fig. 2). Composed of a hydrophilic A block and 


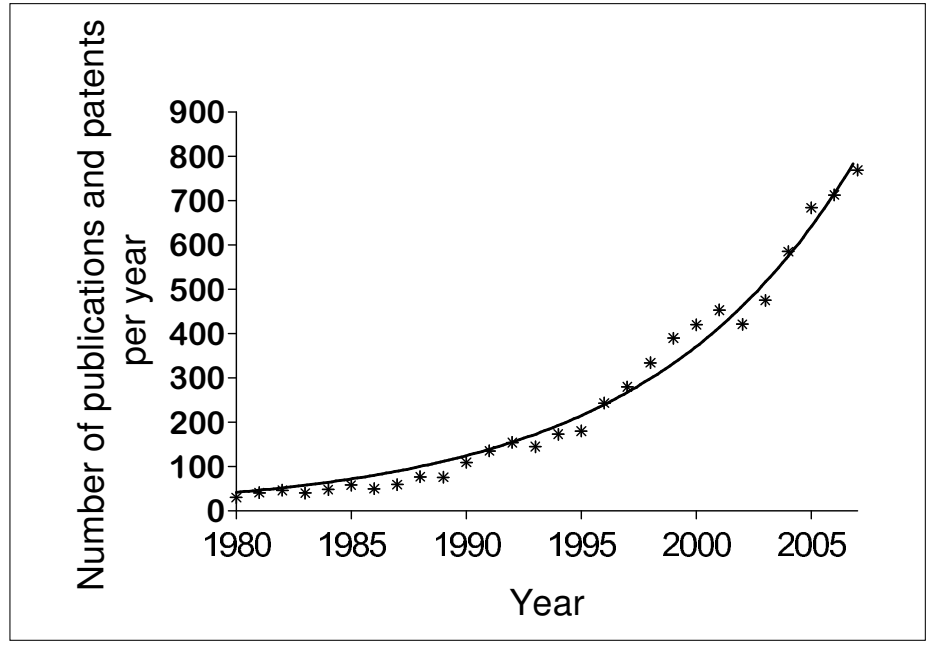

Fig. 1. Number of yearly publications and patents from 1980 to 2007 using the keyword 'polymeric micelles' (source: Scifinder Scholar, February 2008)

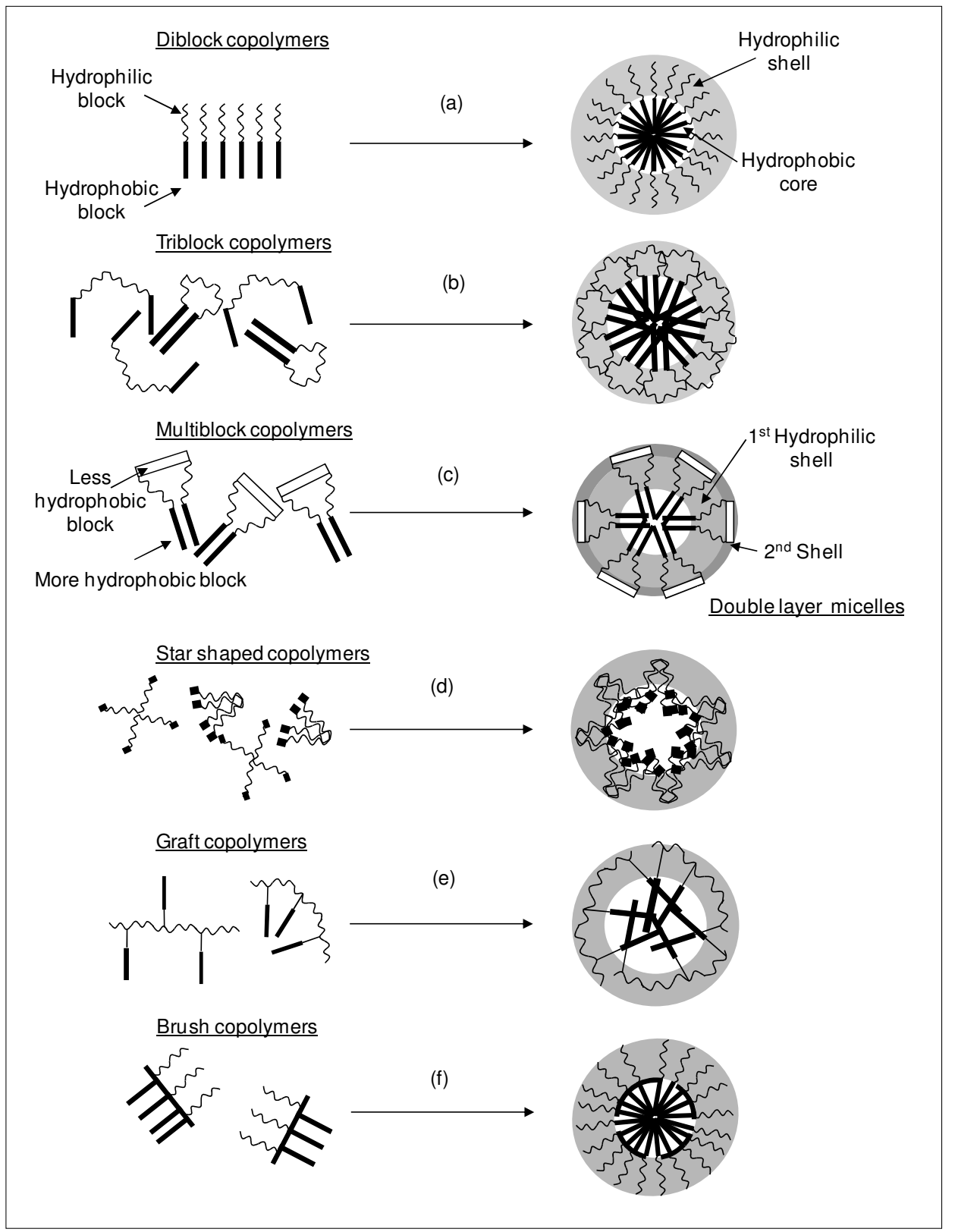

Fig. 2. Different amphiphilic copolymer types and architectures leading to different micelle structures by self-assembling a hydrophobic B block, these copolymers can either be of the linear $\mathrm{AB}$ diblock type, ABA or BAB triblock- or multiblock type-, or a nonlinear composition having more complex architectures like star or branched types. Consequently these copolymers form micelles of different structures.

The self-assembly process is driven by the force to reach the lowest free-energy of the system. ${ }^{[10]}$ In solution the amphiphilic copolymers exist first as unimers until their concentration exceeds the critical micellar concentration (CMC), above which they spontaneously self-assemble into micelles to form colloidal systems of the nano scale. For a given polymer concentration micelles form when the solution temperature reaches the critical micellar temperature (CMT). The lower critical solution temperature (LCST) is the minimum temperature beyond which the polymers self-assemble into micelles. Thus both the CMC and LCST are critical parameters for the existence of stable micelles in the body.

The micelle morphology is controlled mainly by three factors:

i) stretching of the core-forming blocks (free energy of the core),

ii) the surface tension between the coreforming block and the solvent (free energy of the interface), and

iii) repulsion interactions of the coronaforming blocks (free energy of the corona). ${ }^{[10]}$

Different morphologies are possible depending on the characteristics of the amphiphilic polymers (molecular weight, physical state and composition) and on the solution parameters (solvent, polymer concentration, $\mathrm{pH}$, ionic strength, solvent/ co-solvent ratio and others). Amphiphilic diblock copolymers self-assemble into basic shapes like spheres (the predominant form), rods, and wormlike or vesicle structures. When the molecular weight of the hydrophilic block represents more than $50 \%$ of the total polymer molecular weight, spontaneous spherical micelles are formed, whereas with a hydrophilic block slightly below $50 \%$, the micelles take on a wormlike structure. This latter morphology can also be generated by sonication from a copolymer forming initially spherical micelles, as it was shown for poly(ethylene glycol)-b-poly( $\left(\varepsilon\right.$-caprolactone) $\left(\mathrm{PEG}_{5000 \mathrm{~g} / \mathrm{mol}}\right.$ $\left.-\mathrm{PCL}_{6500 \mathrm{~g} / \mathrm{mol}}\right)^{.[11]}$ In degradation studies, wormlike micelles transformed into spherical micelles, when the PCL blocks hydrolyzed and the percentage of hydrophobic blocks dropped to less than $50 \%$ of the overall polymer weight. The physical state of the core forming block also influences the micelle morphology. PEG-poly $(\varepsilon-$ caprolactone-D,L lactide) (PEG-P(CL-DLLA)) with the amorphous poly(D,L-lactide) block self-assembled into spheres, whereas cylindrical structures were formed with the 
crystalline poly(L,L-lactide) block (PEG$\mathrm{P}(\mathrm{CL}-\mathrm{LLA})){ }^{[12]}$ A strong competition between the energy of the crystalline core and the chain stretching corona may explain the formation of the cylindrical shape. Moreover the influence of the composition and the block length has been demonstrated in the following examples; $\mathrm{PEG}_{2000 \mathrm{~g} / \mathrm{mol}}-\mathrm{PCL}_{\mathrm{n}}$ formed a variety of different morphologies with the increase of PCL block length from sphere, rod, wormlike to lamellae, whereas $\mathrm{PEG}_{5000 \mathrm{~g} / \mathrm{mol}^{-}}-\mathrm{PCL}_{\mathrm{n}}$ only self-assembled into spherical micelles. One polymer, $\mathrm{PEG}_{5000 \mathrm{~g} / \mathrm{mol}}-\mathrm{PCL}_{232}$, formed a mixture of spheres and lamellae.[13] PCL also has been used to study the effect of the polymer block-type and -architectures in polymeric micelles (Fig. 2). Classical $\mathrm{PEG}_{5000 \mathrm{~g} / \mathrm{mol}}$ $-\mathrm{PCL}_{\mathrm{n}}$ diblock $^{[13,14]}$ or PCL-PEG-PCL triblock ${ }^{[15]}$ copolymers self-assemble into the common spherical core-shell structure (Fig. 2a and 2b, respectively). BACABtype multiblock copolymers, where $\mathrm{C}$ is a less hydrophobic block than $\mathrm{B}$ as in $\mathrm{PCL}_{\mathrm{n}}$ (PEG-PPO-PEG) ${ }_{1900 \mathrm{~g} / \mathrm{mol}}-\mathrm{PCL}_{\mathrm{n}}$, lead to the formation of a double layer shell-core structure (Fig. 2c). ${ }^{16]}$ Star-shaped copolymers like the four-arm $\left(\mathrm{PEG}_{5000 \mathrm{~g} / \mathrm{mol}^{-}}\right.$ PCL $)_{4}[17]$ or Tetronic ${ }^{\circledR}$-PCL ${ }^{[18]}$ assemble into spherical micelles, wherein the hydrophobic blocks form the core and the hydrophilic PEG chains arrange to form the shell (Fig. 2d). Graft copolymers like PAspg-PCL can form spherical micelles with a shell, in which hydrophilic chains overlap, and entangle (Fig. 2e). ${ }^{[19]} \mathrm{PCL}_{7000 \mathrm{~g} / \mathrm{mol}}$ $-\mathrm{PDMA}_{8000 \mathrm{~g} / \mathrm{mol}}$ brush copolymers with their hydrophobic PCL-backbone form a kind of core-shell-'crosslinked' micelles (Fig. 2f). ${ }^{[20]}$ All of the above-mentioned micelles are characterized by sizes below 100 $\mathrm{nm}$. Yet not all micelle types have proved to be suitable drug carriers.

The influence of the solvent/co-solvent ratio on the micelle shape has been explored by Barghava et al.[21] Using two different solvent systems, DMF/water and DMF/acetonitrile, PEG-polystyrene (PEG-PS) copolymers self-assembled first into spheres and with the decrease of DMF content into cylinders, followed by wormlike, and finally vesicle structures. Mixed morphologies (spheres and cylinders, cylinders and wormlike structures) were visible at intermediate DMF concentrations.

In aqueous media, micelles have a coreshell structure with a hydrophobic dense inner core and a hydrophilic flexible outer shell. It has been shown that many poorly water-soluble drugs can be incorporated efficiently into the hydrophobic core, and thus within the polymeric micelles to become water soluble, facilitated by the hydrophilic micelle shell, which is composed mainly of polyethylene glycol (PEG). Drug loading also influences the micelle morphology. For example a change from spheres to cylinders occurred when indomethacin loading was increased in PEGpoly[2-(diisopropylamino)ethyl methacrylate] (PEG-PDPA) micelles.[22] Further, wormlike micelles from poly(ethylene glycol)-b-poly( $\varepsilon$-caprolactone) $\left(\mathrm{PEG}_{5000 \mathrm{~g} /}\right.$ $\mathrm{mol}^{-} \mathrm{PCL}_{6500 \mathrm{~g} / \mathrm{mol}}$ ) possessed the same diameter as the corresponding spherical ones, but could incorporate twice as much paclitaxel, which is of great interest for an efficient drug loading of the micellar carrier system. ${ }^{[11]}$ Moreover, the stability and cytotoxicity did not differ between the two morphologies.

The presence of PEG, a hydrophilic, water-soluble, nontoxic and non-immunogenic polymer, at the surface of micelles and nanoparticles, respectively enables these nanocarriers to escape from renal exclusion and from opsonization by the mononuclear phagocytic system (MPS). The micelles can protect the incorporated drug from the biological fluids until reaching the target site. With a prolonged circulation time in the blood stream they can accumulate at the inflammation site or in tumor tissues through the enhanced permeability and retention effect (EPR), which resembles a passive targeting. ${ }^{[23]}$ Due to their small size (below $100 \mathrm{~nm}$ ), polymeric micelles can reach the very small vascular vessels and can be internalized into cells more efficiently unlike bigger carriers such as microparticles. ${ }^{[24,25]}$ The release of the incorporated drug at the pathological site can occur by simple diffusion, destabilization of the structure of the micelles by degradation, change in $\mathrm{pH}$ or by other stimuli, which will be discussed in more detail below. There are several other advantages of polymeric micelles in drug delivery. With their low CMC in the micromolar range, polymeric micelles generally have good stability upon dilution. Thus the amount of the polymer surfactant can be reduced in comparison to other classical surfactants in order to formulate a stable drug carrier system. This higher stability of polymeric surfactant-based formulations results in a better shelf-life. The clear appearance and the good injectability of micellar solutions are also advantages for medical treatments. The protection of drugs in the micelle core decreases the adverse side effects observed with free drugs, such as pain and inflammation at the injection site, and systemic side effects, when administered parenterally. ${ }^{[26}$ Propofol is an example of the improvement of a pharmaceutical formulation with polymeric micelles. It is a waterinsoluble anesthetic agent that was found to be more stable in poly(N-vinyl-2 pyrrolidone)-block-poly(D,L-lactide) (PVP-PDLLA) micelle solution than in the currently used water-in-oil emulsion. After reconstitution, the micelle formulation is stable for four days, whereas the standard emulsion can only be used for 6 h. ${ }^{[27]}$ As a second example for advantages of polymeric micelles, a formulation with the potent anticancer drug paclitaxel can be mentioned. Paclitaxel, a chemotherapeutic agent that is almost insoluble in water $(0.3$ $\mu \mathrm{g} / \mathrm{ml})$, is currently marketed as Taxol ${ }^{\circledR}$ and solubilized therein with Cremophor ${ }^{\circledR}$ EL, a polyoxyethylated castor oil. To replace this surfactant, which is known for its toxicity, albumin-bound paclitaxel nanoparticles $\left(\text { Abraxane }{ }^{\circledR}\right)^{[28]}$ and PEGpoly(D,L-lactic acid) polymeric micelles (Genexol ${ }^{\circledR}$ PM) have been developed.[29] These formulations lead to a reduction of severe side effects, thus permitting an increase in the dose during chemotherapy. In vivo studies on human ovarian and breast cancer cells in nude mice demonstrated that tumor growth was reduced $48 \mathrm{~h}$ and 14 days, respectively after administration of Genexol ${ }^{\circledR}$ PM at its maximum tolerated dose (MTD), which is three times higher than in the treatment with Taxol ${ }^{\circledR}$. Moreover, tumor regrowth which was observed for Taxol ${ }^{\circledR[30]}$ is not reported for the Genexol $^{\circledR}$ PM treatment. After one month of treatment, this polymeric micelle formulation showed complete tumor regression.

As mentioned before, drug targeting is a special challenge since the delivery of a drug to the right site of action should be both selective and quantitative. Passive targeting by the EPR effect results from the long-circulation property of micelles. In order to achieve a higher drug dose at the target site, substantial effort has been put into active targeting research. Active targeting can be accomplished by modifying the micelle surface with site-specific ligands, or by creating 'immunomicelles' by attaching monoclonal antibodies. One other strategy for drug targeting uses folate functionalization. Receptors recognizing the vitamin folic acid are over-expressed on many human cancer cells (breast, ovarian, brain, kidney and lung), consequently folate conjugated micelles are preferably recognized and bound on the tumor cells, and can then be internalized by active tumor mechanisms. This mechanism was, for example, observed with adriamycin-loaded folated polymeric micelles. ${ }^{[31]}$ The presence of these ligands on the micelle surface increased the in vitro cytotoxicity by increasing the cellular uptake and the intracellular concentration. The in vivo studies suggested that a defined number of folate groups on the micelles surface showed an optimized carrier-receptor interaction. Tumor treatment with these folated polymeric micelles was more efficient than with the non-folate functionalized micelles or even the free drug. Also, other potent drugs like paclitaxel, ${ }^{[32-34]}$ doxorubicin, ${ }^{[35]}$ and tamoxifen[36] and a multidrug resistance modulator ${ }^{[37]}$ have been formulated with 
folate functionalized micelles and yielded improved results.

A recent paper by Beduneau et al. reviews the different active targeting strategies available for treating brain tumors. ${ }^{[38]}$ One major obstacle for efficient drug delivery to the brain is the blood brain barrier. Different modifications of the surface of nanocarriers (liposomes, micelles or nanoparticles) by grafting endogenous and chimeric ligands, or by directly conjugating proteins and peptides through a covalent or non covalent linkage leads to internalization of the nanocarrier by brain capillary endothelial cells. Other examples of surface functionalization of micelles for drug delivery can be found in the review of Mahmud et al. ${ }^{[39]}$

Application of stimuli responsive micelles is an approach for controlled drug release. When nanocarriers have reached the pathological site, the drug can be released due to destabilization by different stimulations. This can be achieved with external sources like light, ultrasound, hypo- or hyperthermia, or with internal stimuli like $\mathrm{pH}$ change or enzymes. $\mathrm{pH}$-Sensitive micelles have been widely tested in drug delivery, because of increased acidity of tumor and inflammatory tissues compared to healthy tissues. A change in the $\mathrm{pH}$ leads to a demicellization process and the drug is released. It has been reported that doxorubicinloaded $\mathrm{pH}$-sensitive micelles formed from PEG-poly( $\beta$-amino esters) disassembled at pH 6.4 leading to rapid doxorubicin release, whereas at physiological $\mathrm{pH}$ they remained stable. ${ }^{[40]} \mathrm{A}$ higher drug concentration at the pathological site was achieved, resulting in higher anti-tumor efficacy and a higher survival rate in vivo in mice compared to treatment with free drug. In other examples, $\mathrm{pH}$-sensitive polymeric micelles were loaded with adriamycin, ${ }^{[41]}$ paclitaxel $^{[36]}$ or tamoxifen.[36] Other poorly soluble drugs like triclosan, ${ }^{[42]}$ candersartan cilexetil,[43] indomethacin, ${ }^{[4]}$ fenofibrate, ${ }^{[44]}$ and progesterone ${ }^{[44]}$ could be successfully incorporated in similar micelles and resulted in pharmacological improvements.

Thermoresponsive micelles can also be used as controlled release systems. This is particularly the case for poly $(\mathrm{N}$ isopropylacrylamide) (PNIPAAm)-based micelles with a LCST of about $40{ }^{\circ} \mathrm{C}$. ${ }^{45]}$ Below this LCST, AB type copolymers with PNIPAAm as the hydrophilic block form polymeric micelles, which are sterically stabilized by the water-soluble flexible chains of the PNIPAAm block.[46] The lipophilic drug is incorporated in the hydrophobic core and can be stored as a stable formulation. Above the LCST, PNIPAAm becomes insoluble and thus deforms the micelle structure, which results in a rapid diffusion of the drug from the micelles. In a cancer treatment study PNIPAAm-based polymeric micelles are accumulated in a solid tumor site by passive targeting, and upon local heating slightly above the LCST the encapsulated drug was released. $[47,48]$

Ultrasound-sensitive polymeric micelles based on Pluronic ${ }^{\circledR}$ P-105 were developed by the groups of Pitt and Rapoport in the late 1990s. ${ }^{[49]}$ Over time Rapoport and coworkers optimized these micelles by mixing them with PEG-distearoylphosphatidylethanolamine (PEG-DSPE). Mixed micelles demonstrated enhanced stability upon dilution compared to the standard Pluronic ${ }^{\circledR}$ P-105 micelles. Indeed ruboxil, a paramagnetic labelled anthracyclin, showed complete release in very diluted concentrations of fetal bovine serum when incorporated in Pluronic ${ }^{\circledR} \mathrm{P}-105$ micelles, whereas $65 \%$ of the drug was retained in the mixed polymeric micelles. ${ }^{[50]}$ In addition, the treatment of tumors with doxorubicin as the drug was found to be more efficient as indicated by a higher uptake and therefore a decrease of the tumor size, when an ultrasound stimulus was applied to micelles in comparison to non-stimulated micelles. The efficiency of the tumor treatment is even more pronounced when compared to the treatment with the free drug. ${ }^{[50]}$ In summary, polymeric micelles with their specific structure and long circulation in the blood stream are promising carriers for passive and active targeting, especially for cancer treatment.

For the use of amphiphilic polymers in pharmaceutical applications in general, important requirements such as non-toxicity and biocompatibility need to be fulfilled. This constrains the number of available hydrophobic and hydrophilic natural or synthetic polymers.

For the hydrophilic shell-forming block polyethylene glycol (PEG) is the polymer of choice, because of its superior properties, and its approval by the Food and Drug Administration (FDA). Despite its non-degradability PEG is non-toxic and can easily be removed from the body through the excretion pathways, as long as the molecular weight is less than $15 \mathrm{kDa}$. Thus PEG with molecular weights between 2 and 15 $\mathrm{kDa}$ are suitable in polymeric micelles for drug delivery. One of the main advantages resides in the efficient protection of incorporated drugs in pegylated nanocarriers. [51] Other polymers like poly(N-vinyl-2pyrrolidone) (PVP), ${ }^{[52,53]}$ polyvinyl alcohol (PVA) and its derivatives, ${ }^{[54]}$ or recently studied poly(ethyl ethylene phosphate) (PEEP) ${ }^{[55]}$ could be alternatives to PEG. PVP a non-ionic, biocompatible and watersoluble synthetic polymer is often preferred to PEG for freeze drying formulations, because of its better cryoprotectant properties. [56] Polyphosphates were recently investigated as polymers for micellar systems, ${ }^{[55]}$ because of their biocompatibility, degradability and pendant chain functionality, but further work is needed for a proof of concept.

For the hydrophobic core-forming block, a large number of polymers meet the requirements for pharmaceutical applications. Here ionic or non-ionic, degradable or nondegradable polymers can be considered. In polyion complex micelles (PICM) a charged core-forming block can more efficiently incorporate negatively charge drugs like plasmid DNA, oligodeoxyribonucleotides, some polysaccharides, enzymes or photosensitizers. The typical positively charged polymers are poly(ethylenimines) (PEI), [57] polyacrylamides, ${ }^{[58,59]}$ poly(L-lysine $)^{[60]}$ or poly(2(N,N-dimethylamino)ethyl methacrylate) (PDMAEMA). [61] In contrast, polyanionic polymers, like poly(methacrylic acid) (PMAA) ${ }^{[47]}$ or poly(aspartic acid) (PAsp), ${ }^{[62]}$ are used for the incorporation of polycationic peptides or lipids. ${ }^{[63]}$

Various non-ionic hydrophobic corebuilding degradable and non-degradable polymers have been investigated. Here we will focus on the possible polymers from three different classes including polyethers, poly(L-amino acids) and polyesters. Certainly there are other important biodegradable polymers, like polyanhydrides or polyurethanes, but to date they are used more as implants, microspheres, discs or other matrices for localized drug delivery. ${ }^{[64,65]} \mathrm{A}$ few examples of polymeric micelles made from oligoanhydrides and PEG have been reported by Najafi et al., who demonstrated the ability to incorporate a hydrophilic model drug, calcein. ${ }^{[66]}$

The most interesting polyethers for micellar drug delivery systems are the triblock copolymers (ABA type) of PEG and poly(propylene oxide) (PPO). They are marketed and commonly known as Pluronics $^{\circledR}$ (BASF) ${ }^{[67]}$ or as poloxamers, their non-proprietary name. ${ }^{[68]}$ Depending on the compositions and block-lengths PEG-PPO multiblock copolymers show interesting material properties, including the formation of micellar structures or temperaturedependent gels. For example Pluronic ${ }^{\circledR}$ based micelles were studied by Gao et al. ${ }^{[50]}$ and Exner et al. ${ }^{[69]}$ for cancer therapy. In these studies, doxorubicin and carboplatin, respectively were successfully incorporated into the micelles. For doxorubicin-loaded Pluronic ${ }^{\circledR}$ micelles high drug uptake could be achieved in multidrug resistant cell lines through ultrasonic irradiation of the tumor. ${ }^{[50]}$ An efficient tumor decrease was observed using this method.

Derived from natural L-amino acids, poly(L-amino acids) are very interesting biocompatible polymers and were studied as possible hydrophobic core-forming blocks. ${ }^{[70]}$ Several poly(L-amino acid)based micelles have been investigated as $\mathrm{pH}$-sensitive polymeric drug carriers. At acidic $\mathrm{pH}$ in the tumor, protonation of free 
amine and carboxyl groups in the polymer chains leads to a destabilization of the micelle structure and resulted in the controlled release of drug. ${ }^{\text {[71] }}$ PEG-poly(Lhistidine) micelles loaded with doxorubicin generated a decrease of human ovarian carcinoma subcutaneously xenografted in mice. ${ }^{[72]}$ As another example PEG-poly(Llysine) and PEG-poly(L-ornithin) micelles were studied for DNA delivery. ${ }^{[73]} \mathrm{A}$ higher condensation of DNA and a higher DNA transfection of two mammalian cell lines in vitro were achieved with these micelle carriers compared to the parent homopolymers, poly(L-lysine) or poly(L-ornithine) Disadvantageously the higher haemolytic activity could limit the intravenous use of these novel vesicles.

As an example for non-pH sensitive poly(L-amino acids) polymeric micelles, benzyl ester of PEG-poly(aspartic acid) (PEG-P(Asp)) micelles yielded a higher drug loading of the water-insoluble anticancer agent camptothecin and better stability compared to other esterified copolymers and to free drug. ${ }^{[74]}$ In these studies mice, transplanted with colon solid tumor cells, and treated with benzylester-PEG-P(Asp) micelles, had a longer circulation time, an increased accumulation in the tumor, and thus a more efficient antitumor activity.[23] Several hydrophobic core-forming poly(Lamino acids) could improve micelle stability and showed promising results for cancer or gene therapy applications.

Poly(hydroxyalkanoic acids) are the most commonly used polymers in PEGpolyester micelles for drug delivery, because of their outstanding biodegradability and biocompatibility. Due to the vast number of published articles on PEG-polyester micelles we can only present some selected examples of recent achievements, and would like to refer the readers to other more detailed review articles on polymeric micelles. ${ }^{[5,6,75,76]}$

Poly(butyrolactone) (PBL) is preferentially used in its $\beta$ form, and is derived from the natural production in microorganisms. $\beta$-PBL is characterized by a higher hydrophobicity and crystallinity compared to the synthetic $\alpha$-PBL. Despite a good stability, a low CMC and small size, the potential of PEG-PBL-PEG triblock micelles (ABA type) was limited due to the slow biodegradation of the PBL blocks in vivo. ${ }^{[77]}$ Contrary the PBL-PEG-PBL copolymers, BAB type, showed a faster biodegradation rate, while having similar sizes and CMC values. ${ }^{[78]}$ However, the incorporation of hydrophobic drugs needs to confirm their feasibility for drug delivery, even if pyrene as a model compound was incorporated with success.

Different studies on the semi-crystalline poly(valerolactone) (PVL) as the hydrophobic core-forming block were reported. ${ }^{[79-81]}$ As one example, $\mathrm{PEG}_{2000 \mathrm{~g} / \mathrm{mol}^{-}}$
$\mathrm{PVL}_{2000 \mathrm{~g} / \mathrm{mol}}$ was used for the formulation of paclitaxel.[81] With these micelles the water solubility was increased to $9 \mathrm{mg} / \mathrm{ml}$. When compared to the actual paclitaxel solubilization of $6 \mathrm{mg} / \mathrm{ml}$ in the mixture Cremophor ${ }^{\circledR}$ EL/dehydrated alcohol in Taxol ${ }^{\circledR}$, the result suggests that a lower amount of PEG-PVL surfactant would be needed to achieve the same dose. In vivo studies are required to show that the slow degradation of PVL and the expected longer elimination time from the body will not be a problem.

Poly(caprolactone) (PCL) is another very suitable polymer for the core-forming block, because of its proven biocompatible properties, therefore PEG-PCL diblock copolymers have commonly been investigated for drug delivery. Recently, protoporphyrin IX, a hydrophobic photosensitizer, was incorporated into $\mathrm{PEG}_{5000 \mathrm{~g} / \mathrm{mol}}-\mathrm{PCL}_{4100 \mathrm{~g} / \mathrm{mol}}$ micelles, and a higher cellular uptake and higher photocytotoxicity was obtained compared to the free photosensitizer. ${ }^{[82]}$ In another example reported by Aliabadi et al., cyclosporine A, an immunosuppressive agent, was encapsulated in $\mathrm{PEG}_{5000 \mathrm{~g} / \mathrm{mol}}$ $-\mathrm{PCL}_{13000 \mathrm{~g} / \mathrm{mol}}$ micelles. ${ }^{[83]}$ Here the drug solubility was increased 80-fold compared to its water solubility. ${ }^{[84]}$ High drug loadings in these micelles envision the possible replacement of other surfactants, like Cremophor ${ }^{\circledR} \mathrm{EL}$ in the current formulation Sandimmun ${ }^{\circledR}$, which shows severe side effects. In studies for cancer treatment PEG-PCL polymeric micelles were loaded with chemotherapeutic agents like cisplatin, ${ }^{[20]}$ doxorubicin, ${ }^{[85,86]}$ paclitaxel ${ }^{[87]}$ and recently tested newer compounds such as $\beta$-lapachone ${ }^{[86]}$ and curcumin. ${ }^{[88,89]}$ Other approaches focus on specific-specific targeting PEG-PCL carriers functionalized with folic acid, heparin or epidermal growth factor (EGF) for the release of a chemotherapeutics like paclitaxel, ${ }^{[32]}$ of both indomethacin and basic fibroblast growth factor (bFGF) ${ }^{[90]}$ or ellipticine. ${ }^{[91]}$ Higher internalization and higher cytotoxicity were observed, and thus improved significantly the tumor treatment compared to the nonfunctionalized micelles.

Among the various poly(hydroxy-alkanoic acids), poly(lactic acid) (PLA) and poly(lactic-co-glycolic acid) (PLGA) copolymers are the most outstanding polyesters in drug delivery applications. Environmental friendliness, synthesis from renewable resources, biodegradability into non-toxic lactic acid, and excellent biocompatibility have made it the polymer of first choice for many medical applications. Available stereochemically different polylactides are characterized by different physical properties. The racemic D,L-PLA is amorphous, whereas the enantiomeric pure $\mathrm{L}^{-}$or $\mathrm{D}$ PLA (PLLA and PDLA, respectively) are crystalline materials, resulting in different biodegradation times. Poly(lactide-glycolic acid) (PLGA), which is a PLA functionalized with glycolic acid units or blocks in the polymer chain, degrades faster than pure PLA and is often used to tailor the degradation time of PLA drug carrier systems. Therefore, depending on the polymer used, these PLA-based micelles have significantly different properties. $\mathrm{PEG}_{\mathrm{n}}-\mathrm{P}(\mathrm{CL}$ DLLA) $8121 \mathrm{~g} / \mathrm{mol}$ had a lower CMC than $\mathrm{PEG}_{\mathrm{n}}-\mathrm{P}(\mathrm{CL}-\mathrm{LLA})_{6614 \mathrm{~g} / \mathrm{mol}}$, and both $\mathrm{mi}-$ celles adopted different morphologies. ${ }^{12]}$ Mixing PEG-PLLA and PEG-PDLA copolymer led to a stereocomplex formation of the PLA chains in the micelle core, which had better micelle stability with a lower CMC, smaller size, and higher incorporation efficacy of rifambin, than PEG-PLLA or PEG-PDLA micelles. ${ }^{\text {[92] }}$ In Genexol$\mathrm{PM}^{\circledR}$, PEG-PDLLA micelles are used as the drug carrier for paclitaxel.[30] A multicenter Phase II trial for the treatment of advanced non-small-cell lung cancer in combination with cisplatin ${ }^{[29]}$ has been successfully finished, and the same formulation has entered into a Phase II study for advanced pancreatic cancer treatment. ${ }^{[93]}$ Other drugs like 5-fluoroacil,[94] doxorubicin, ${ }^{[86,95,96]} \beta$-lapachone, ${ }^{[86,97]}$ campthothecin ${ }^{[98]}$ and amphotericin $\mathrm{B}^{[99]}$ have been incorporated into PEG-PLA micelles, and different polymer architectures like linear or star-branched, diblock or triblock polymers, homopolymer or mixed polymer micelles were investigated. Star-branched polymeric micelles showed no difference in comparison to linear polymeric micelles for 5- fluoroacil release, whereas paclitaxel was released more rapidly and completely. [94] In mixed micelles with doxorubicinloaded poly(N-isopropylacrylamide-comethacrylicacid)-graft-poly(D,L-lactide) (P(NIPAAm-co-MAAc)-g-PDLLA) the PEG-PLA did not affect the $\mathrm{pH}-$ and thermoresponsive properties, but advantageously prevented the adsorption of albumin, increasing the circulation time in the blood stream. ${ }^{[96]}$ PLA-PEG-PLA triblock copolymers with acryl end groups self-assembled into micelles, which were transformed into nanogels by UV irradiation. ${ }^{\left[{ }^{8}\right]}$ Loaded with campthothecin these nanogels showed better stability and a sustained release for at least 20 days in contrast to complete release for non-irradiated micelles after few hours. Hydrophilic shell-forming blocks other than PEG have been explored for PLA- or PLGA-based copolymer micelles. Recently PLA-poly(L-glutamic acid) micelles were developed for magnetic resonance imaging (MRI) diagnostic systems. ${ }^{[100]}$ Gadolinium $(\mathrm{Gd})$ ions chelated with DTBA were incorporated into these micelles as an MRI probe. They were stable upon dilution and showed a two times higher relaxivity compared to the free DTBA-Gd complex. Further in $v i$ vo studies should evaluate the feasibility of these micellar diagnostic systems for their 
application in patients. Poly(L-lysine) could be another alternative polymer to PEG as the shell-building block. Grafted on PLGA, the resulting amphiphilic polymer selfassembled into micelles, which showed lower cytotoxicity and higher transfection efficiency than poly(L-lysine) itself, and could be a possible carrier for gene delivery.[101] In comparison to PEG, these poly(L-amino acid)-based micelles are entirely biodegradable. Stimuli responsive micelles based on PEG-poly(L-histidine) (PEG-PHis) copolymer micelles were also investigated. Upon a change of $\mathrm{pH}$ in tumor tissue, the imidazole side groups along the poly(L-histidine) backbone are ionized $\left(\mathrm{pH}<\mathrm{pK}_{\mathrm{b}}\right)$, transforming the hydrophobic PHis-block into an hydrophilic water-soluble one. This is accompanied by swelling of the micelles and concurrent drug release. ${ }^{[102]}$ Loaded with doxorubicin PLA-PEG-PHis micelles showed an effective treatment of human breast tumors. ${ }^{[95]}$ Thermoresponsive micelles made of PLAbased polymers were studied with (PNIPAAm-co-DMAAm)-PLGA copolymers for the delivery of chemotherapeutic agents like paclitaxel[103] and doxorubicin. ${ }^{[59]}$ These micelles showed a higher drug release upon thermo-stimulation above the $\operatorname{LCST}\left(39.5^{\circ} \mathrm{C}\right)$ and a higher cytotoxicity on tumor cells compared to free drug. As an example for tumor targeting micelles, folate conjugated PEG-PLGA micelles were studied by Yoo et al. ${ }^{[104]}$ and Zhao et al. ${ }^{[35]} \mathrm{A}$ higher uptake, higher cytotoxicity, and higher apoptosis were obtained with these folate-functionalized micelles compared to the non-folated micelles and the free drug, respectively. [35]

PEG-polyester micelles are potent drug carriers compared to many classical surfactant-based micelles. A higher stability in diluted solutions due to their lower CMC, PEG copolymer micelles have long circulation properties and can be functionalized as targeting or as stimuli responsive drug release carrier systems. An increased accumulation, a higher drug uptake by the tumor or inflammation cells accompanied with a more effective tumor treatment can often be achieved with polymeric micelles. Regarding this, albeit an incomplete list, PCL, PLA and PLGA are the most promising polyesters. Because of their approval by the FDA for drug products in human use, new formulations with these pegylated excipients might lead easier and faster to new applicable therapeutics.

\section{Recent Developments of Hydrophobic Substituted PLA- based Polymeric Micelles}

Despite the outstanding applications of PLA in medical applications, limitations for the incorporation of hydrophobic drugs for pharmaceutical formulations are often encountered. Since a more hydrophobic core is desirable for better incorporation into PLA-based polymeric micelles, we focused in our own research efforts on the controlled functionalization of PLA with hydrophobic substituents. Therefore methyl groups along the PLA polymer backbone were substituted by hexyl groups leading to the more hydrophobic hexyl-substituted poly(lactides) (hexPLA). ${ }^{105]}$ PEG-hexPLA copolymers formed micelles in aqueous solutions and incorporated hydrophobic drugs, e.g. griseofulvin, more efficiently than comparable PEG-PLA micelles. ${ }^{[106]}$ Recently we investigated the potential of these novel PEG-hexPLA micelles for possible use in photodynamic therapy (PDT) in cancer treatment. Initial results in incorporating the hydrophobic photosensitizer meso-tetra( $p$-hydroxyphenyl)porphine (THPP) (Fig. 3), and stability studies of THPP-loaded PEG-hexPLA micelles will be presented.

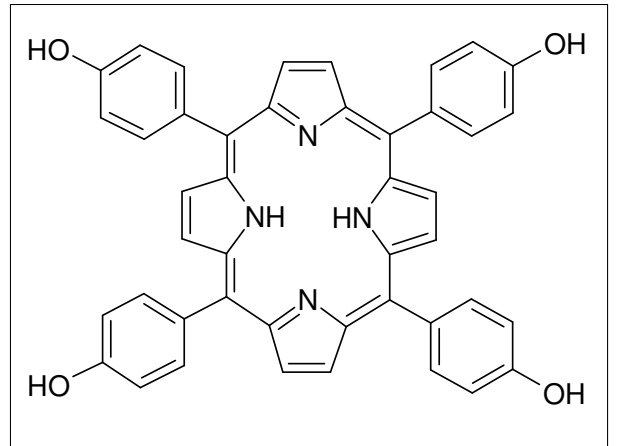

Fig. 3. Molecular structure of meso-tetra(phydroxyphenyl)porphine (THPP)

In order to compare PEG-monohexPLA and PEG-dihexPLA micelles ${ }^{[107]}$ for their increased hydrophobicity and a possible higher incorporation rate of THPP, a comparable PEG-'standard' poly(D,L-lactide) (PEG-PDLLA) was used as a control. All three copolymers were of comparable molecular weights of around $5200 \mathrm{~g} / \mathrm{mol}$ and a comparable polydispersity of 1.2 . The PEG-(hex)PLA polymeric micelles were prepared by the co-solvent evaporation technique. Both the drug and the copolymer were dissolved in the water-miscible organic solvent acetone or THF/acetone $(1: 1)$. After the dropwise addition of the organic solution into water under sonication, accompanied by the self-assembly to micelles, the organic solvent was slowly evaporated. After equilibrium overnight of the remaining aqueous solution the micelles were characterized by their sizes, morphologies and drug incorporation. Unloaded PEG-(hex)PLA micelles had sizes between 18-27 nm, whereas THPPloaded micelles were only slightly larger with $21-45 \mathrm{~nm}$. Thus the loaded micelle sizes remained below $50 \mathrm{~nm}$, a preferred 'sub-100 nm' size, facilitating good internalization into tumor cells by the EPR effect. Dynamic light scattering at different detection angles confirmed a unimodal size distribution for loaded and unloaded PEG-hexPLA and PEG-PLA micelles. The micelles sizes and morphologies were confirmed by TEM measurements. As shown in Fig. 4 unloaded and loaded PLAbased polymeric micelles had comparable sizes with spherical shapes. A study of polymeric micelle solutions stored at room temperature for 10 months proved their stability and the long shelf-life of this formulation. The amount of THPP in the diverse hexPLA- and PLA-based micelles was assessed by UV spectroscopy. The results for THPP incorporation into these micelles are presented in Fig. 5. Shown is the actual achieved loading in dependence of the intended loading, which is based on the drug concentration in the organic solution used for the micelle preparation. Furthermore, the two lines indicate a theoretical 80 and $100 \%$ incorporation, demonstrating the efficiency of the THPP incorporation. THPP incorporation of micelles prepared with acetone and with THF/acetone, respectively, as solvent systems are presented. For intended small loadings up to $50 \mathrm{mg}$ THPP/g copolymer no significant differences were observed between the three different polymers and the organic solvents used. Incorporation rates were at least $80 \%$. For a desired higher drug loading of $100 \mathrm{mg}$ THPP/g copolymer and 300 $\mathrm{mg} \mathrm{THPP} / \mathrm{g}$ copolymer, respectively better incorporation rates were obtained when using a polymer/drug solution in the THF/ acetone $(1: 1)$ mixture, which facilitates drug solubility. It should be noted that in both cases the incorporation was higher and much more efficient with the novel PEGhexPLA micelles than with the standard PEG-PLA micelles. The highest obtained drug loading was $123 \mathrm{mg}$ THPP/g PEGdihexPLA, although with less efficiency $(41 \%)$ compared to 96 and $88 \mathrm{mg}$ THPP for the dihexPLA and the monohexPLA, respectively, which lead to around $90 \%$ efficiency for the intended lower drug loading. Since the molecular weights and ratios of PEG/hexPLA are not optimized, it is expected that the loading capacity for THPP could be higher. Nevertheless the actual results already show a prominent increased water solubility of THPP in PEG-hexPLA micelles. Within the PEGdihexPLA micelles $480 \mathrm{mg}$ THPP/L water could be dissolved compared to only $2.2 \mathrm{mg} / \mathrm{l}$ in pure water, which corresponds to an increase of 218 fold! As mentioned, this result still stands for non-optimized conditions, thus further developments in PEG-hexPLA micellar formulations for 

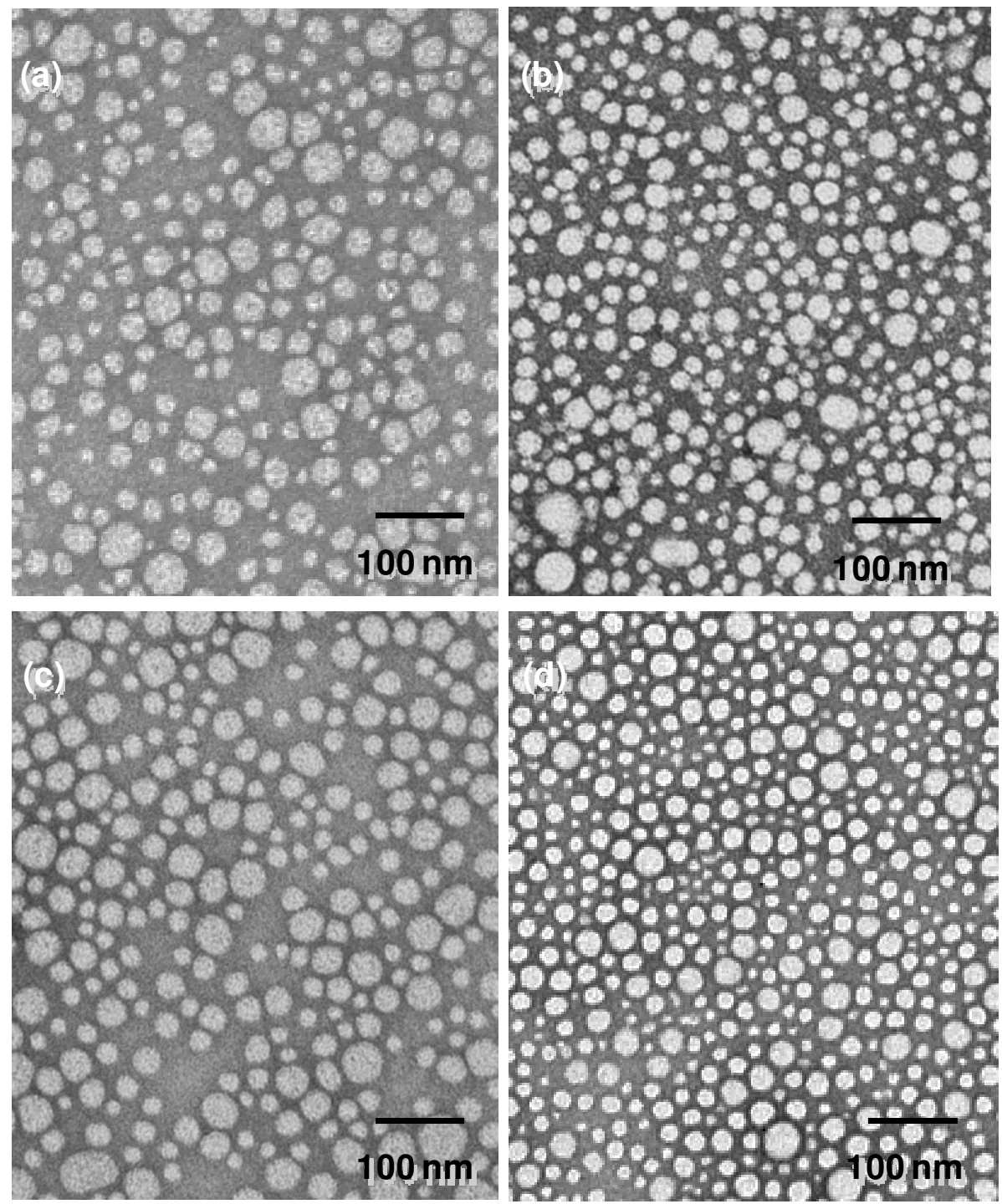

Fig. 4. TEM images of unloaded (a) and THPP-loaded PEG-monohexPLA (b) micelles, and for comparison unloaded PEG-dihexPLA (c) and PEG-PLA (d) micelles

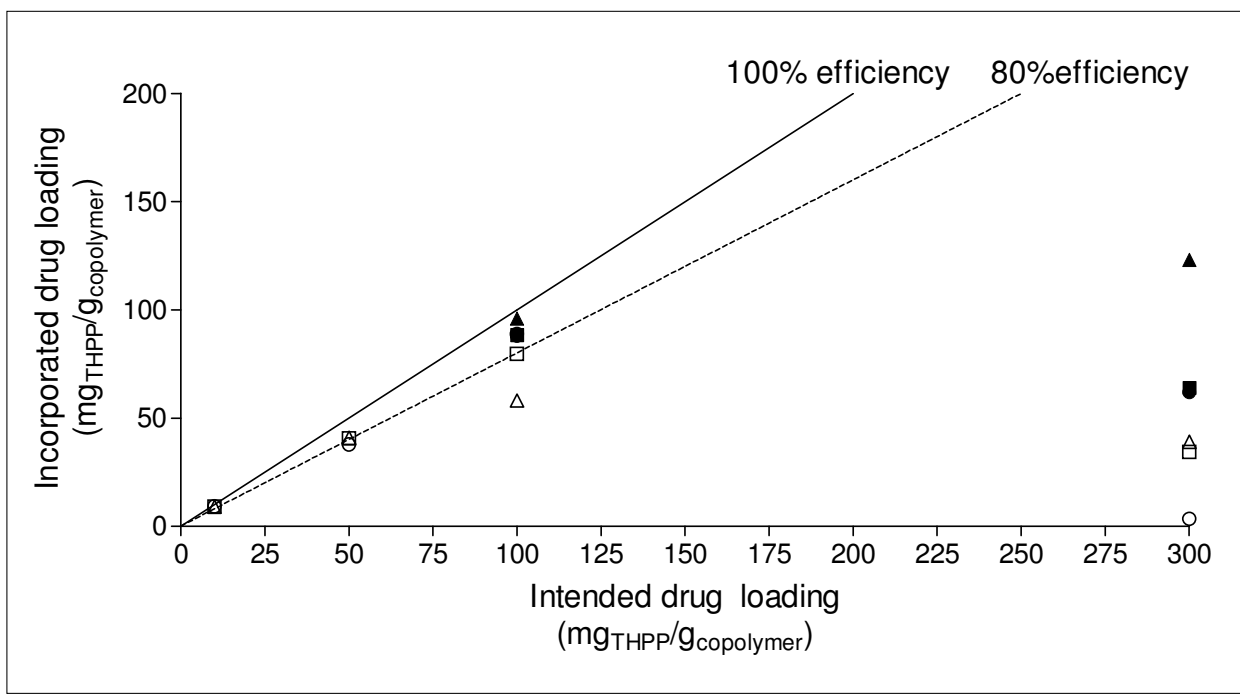

Fig. 5. Incorporation of THPP in PEG-PLA $(O, \bullet)$, PEG-monohexPLA $(\square, \boldsymbol{\square})$ and PEG-dihexPLA $(\triangle, \mathbf{A})$ micelles in function of the intended drug loading for the two preparation methods, with acetone (empty symbol) and THF/acetone (plain symbol), respectively
THPP delivery could lead to a promising alternative for PDT applications in cancer treatment. The increased THPP drug loadings within the hexyl-substituted PLAbased micelles in comparison to standard PLA validate our strategy of improving hydrophobic drug formulations with 'hydrophobized' PLA. As reported in our previous papers the hexPLA polymers degrade by hydrolysis to non-toxic lactic acid and 2-hydroxyoctanoic acid, which has already been approved for topical applications, and which is an important aspect for the feasibility of these polymers for medical applications. Therefore, on-going studies focus on the toxicity issues of these novel PEGhexPLA micelles. Initial cell culture- and human blood toxicity tests show positive results and will soon be presented.

\section{Summary}

Polymeric micelles as colloidal drug delivery systems have attracted great interest in the last 15 years due to their favorable properties. Formed by the self-assembling of amphiphilic copolymers, polymeric micelles have a core-shell structure which can adopt several morphologies. The protective hydrophilic shell offers the micelles long circulation times in the blood stream, and a low CMC a high stability upon dilution. This can enable micelles to accumulate in tumors by passive targeting and the EPR effect. Active targeting to the site of action can be achieved by binding specific ligands or antibodies on micelle surfaces. The use of stimulus-responsive polymeric micelles can enhance the controlled drug release. For drug delivery, PEG is the most outstanding and widely used hydrophilic polymer for the micelle shell. Considerations regarding toxicity and biocompatibility limit the choice of numerous hydrophobic polymers for the core-forming block. Next to several polyethers, poly (Lamino acids), polyesters, particularly PLA have distinguished properties for medical applications. Nevertheless, the incorporation of hydrophobic drugs into PLA micelles often has limitations. In order to increase the hydrophobicity along the PLA backbone for better incorporation of hydrophobic drugs we followed the strategy to substitute the methyl with more hydrophobic hexyl side groups. Hexyl-substituted lactides copolymerized with PEG lead to amphiphilic copolymers, which self-assemble in aqueous solution into homogeneous spherical micelles ranging in sizes from 20 to $45 \mathrm{~nm}$. These PEG-hexPLA micelle formulations showed 10 months shelf stability at room temperature. The incorporation of a poorly water-soluble drug, like the photosensitizer THPP, did not affect the micelle size or the micelle 
morphology. However, by incorporation into PEG-hexPLA micelles the water solubility was increased more than 200 times compared to the water solubility of the free drug. These initial results suggest that the use of PEG-hexyl-substituted polylactides micelles as drug delivery carriers for photodynamic therapy is feasible.

\section{Abbreviations}

bFGF: basic fibroblast growth factor

CL: $\varepsilon$-caprolactone

CMC: critical micellar concentration

CMT: critical micellar temperature

dihexPLA: di-hexyl-substituted polylactides DLLA: D,L-lactide

D-PLA: poly(D-lactide)

D,L-PLA: poly(D,L-lactide)

DMAAm: N,N-dimethylacrylamide

DMF: dimethylformamide

DSPE: distearoylphosphatidyletha-

nolamine

DTBA: $p$-aminobenzyldiethylenetriamine penta(acetic acid)

EGF: epidermal growth factor

EPR: enhanced permeation and retention

effect

FDA: Food and Drug Administration

Gd: gadolinium

hexPLA: hexyl-substituted poly(lactide)

(hex)PLA: hexyl-substituted poly(lactide)

and poly(lactide), respectively

LCST: lower critical solution temperature

LLA: L,L-lactide

L-PLA: poly(L-lactide)

MAAc: methacrylic acid

monohexPLA: mono-hexyl-substituted

poly(lactide)

MPS: mononuclear phagocytic system

MRI: magnetic resonance imaging

MTD: maximum tolerated dose

PAsp: poly(aspartic acid)

PBL: poly(butyrolactone)

PCL: $\operatorname{poly}(\varepsilon$-caprolactone)

PDLA: poly(D-lactide)

PDLLA: poly(D,L-lactide)

PDMA: poly[2-(N,N-dimethylamino)ethyl

methacrylate]

PDMAEMA: poly(2-( $N, N$-dimethylamino) ethyl methacrylate)

PDPA: poly[2-(diisopropylamino)ethyl

methacrylate]

PDT: photodynamic therapy

PEEP: poly(ethyl ethylene phosphate)

PEG: poly(ethylene glycol)

PEI: poly(ethylene imines)

PG: poly(L-glutamic acid)

PHis: poly(L-histidine)

PICM: polyion complex micelles

PLA: poly(lactic acid) or poly(lactide)

PLGA: poly(lactic-co-glycolic acid)

PLLA: poly(L-lactide)

PMMA: poly(methacrylic acid)

PNIPAAm: poly(N-isopropylacrylamide)

PPO: poly(propylene oxide)

PS: poly(styrene)

PVA: poly(vinyl alcohol)
PVL: poly(valerolactone)

PVP: poly(N-vinyl-2-pyrrolidone)

THF: tetrahydrofuran

THPP: $m e s o$-tetra( $p$-hydroxyphenyl)porphine

$$
\text { Received: July 11, } 2008
$$

[1] O. M. Koo, I. Rubinstein, H. Onyuksel, Nanomedicine: NBM 2005, 1, 193.

[2] Search with Scifinder Scholar 2006 with the keyword 'polymeric micelles' after refinement by Document Type 'Journal, Review and Patent' and analysis by Publication Year, 2008.

[3] V. Torchilin, Adv. Drug Deliv. Rev. 2002, 54, 235.

[4] V. Torchilin, Pharm. Res. 2007, 24, 1.

[5] G. Gaucher, M. H. Dufresne, V. P. Sant, N. Kang, D. Maysinger, J. C. Leroux, $J$. Control. Release 2005, 109, 169.

[6] D. Sutton, N. Nasongkla, E. Blanco, J. Gao, Pharm. Res. 2007, 24, 1029.

[7] D. Le Garrec, M. Ranger, J. C. Leroux, Am. J. Drug Deliv. 2004, 2, 15.

[8] N. Rapoport, Prog. Polym. Sci. 2007, 32, 962.

[9] N. Nishiyama, K. Kataoka, Pharmacol. Ther. 2006, 112, 630.

[10] L. Zhang, A. Eisenberg, Polym. Advan Technol. 1998, 9, 677.

[11] S. Cai, K. Vijayan, D. Cheng, E. Lima, D. Discher, Pharm. Res. 2007, 24, 2099.

[12] J. Zhang, L. Q. Wang, H. Wang, K. Tu, Biomacromolecules 2006, 7, 2492.

[13] Z. X. Du, J. T. Xu, Z. Q. Fan, Macromolecules 2007, 40, 7633.

[14] I. L. Gyun Shin, S. Yeon Kim, Y. Moo Lee, C. Soo Cho, K. S. Yong, J. Control. Release 1998, 51, 1 .

[15] L. Piao, Z. Dai, M. Deng, X. Chen, X. Jing, Polymer 2003, 44, 2025.

[16] C. Liu, C. Gong, Y. Pan, Y. Zhang, J. Wang, M. Huang, Y. Wang, K. Wang, M. Gou, M. Tu, Y. Wei, Z. Qian, Colloid. Surface A 2007, 302, 430.

[17] C. Lu, L. Liu, S. R. Guo, Y. Zhang, Z. Li, J. Gu, Eur. Polym. J. 2007, 43, 1857.

[18] J. S. Lee, D. H. Go, J. W. Bae, S. J. Lee, K. D. Park, J. Control. Release 2007, 117 , 204.

[19] J. H. Jeong, H. S. Kang, S. R. Yang, K. Park, J. D. Kim, Colloid. Surface A $\mathbf{2 0 0 5}$ 264, 187.

[20] P. Xu, E. A. Van Kirk, S. Li, W. J. Murdoch, J. Ren, M. D. Hussain, M. Radosz, Y. Shen, Colloid. Surface B 2006, 48, 50.

[21] P. Bhargava, J. X. Zheng, P. Li, R. P. Quirk, F. W. Harris, S. Z. D. Cheng, Macromolecules 2006, 39, 4880 .

[22] C. Giacomelli, V. Schmidt, R. Borsali, Langmuir 2007, 23, 6947.

[23] K. Kawano, M. Watanabe, T. Yamamoto, M. Yokoyama, P. Opanasopit, T. Okano, Y. Maitani, J. Control. Release 2006, 112 329.

[24] S. McClean, E. Prosser, E. Meehan, D O'Malley, N. Clarke, Z. Ramtoola, D. Brayden, Eur. J. Pharm. Sci. 1998, 6, 153.

[25] M. P. Desai, V. Labhasetwar, E. Walter, R. J. Levy, G. L. Amidon, Pharm. Res. 1997, 14, 1568.

[26] P. Picard, M. R. Tramer, Anesth. Analg. 2000, 90, 963
[27] F. Ravenelle, S. Gori, D. Le Garrec, D. Lessard, L. Luo, D. Palusova, J. Sneyd, D. Smith, Pharm. Res. 2008, 101, 186.

[28] N. Desai, V. Trieu, Z. Yao, L. Louie, S. Ci, A. Yang, C. Tao, T. De, B. Beals, D. Dykes, P. Noker, R. Yao, E. Labao, M. Hawkins, P. Soon-Shiong, Clin. Cancer Res. 2006, 12, 1317.

[29] D. W. Kim, S. Y. Kim, H. K. Kim, S. W. Kim, S. W. Shin, J. S. Kim, K. Park, M. Y. Lee, D. S. Heo, Ann. Oncol. 2007, 18, 2009.

[30] S. C. Kim, D. W. Kim, Y. H. Shim, J. S. Bang, H. S. Oh, S. W. Kim, M. H. Seo, J. Control. Release 2001, 72, 191.

[31] Y. Bae, N. Nishiyama, K. Kataoka, Bioconjugate. Chem. 2007, 18, 1131.

[32] E. K. Park, S. Y. Kim, S. B. Lee, Y. M. Lee, J. Control. Release 2005, 109, 158.

[33] W. Y. Seow, J. M. Xue, Y. Y. Yang, Biomaterials 2007, 28, 1730

[34] Y. Wang, L. Yu, L. Han, X. Sha, X. Fang, Int. J. Pharm. 2007, 337, 63.

[35] H. Zhao, L. Y. L. Yung, Int. J. Pharm. 2008, 349, 256.

[36] M. Licciardi, G. Giammona, J. Du, S. P. Armes, Y. Tang, A. L. Lewis, Polymer 2006, 47, 2946.

[37] X. Yang, W. Deng, L. Fu, E. Blanco, J. Gao, D. Quan, X. Shuai, J. Biomed. Mater. Res. A 2007, 86, 48 .

[38] A. Beduneau, P. Saulnier, J. P. Benoit, Biomaterials 2007, 28, 4947.

[39] A. Mahmud, X. B. Xiong, H. M. Aliabadi, A. Lavasanifar, J. Drug Target. 2007, 15 , 553.

[40] J. Ko, K. Park, Y. S. Kim, M. S. Kim, J. K. Han, K. Kim, R. W. Park, I. S. Kim, H. K Song, D. S. Lee, I. C. Kwon, J. Control. Release 2007, 123, 109

[41] Y. Bae, N. Nishiyama, S. Fukushima, H. Koyama, M. Yasuhiro, K. Kataoka, Bioconjugate. Chem. 2005, 16, 122.

[42] D. A. Chiappetta, J. Degrossi, S. Teves, M. D'Aquino, C. Bregni, A. Sosnik, Eur. J. Pharm. Biopharm. 2008, 69, 535.

[43] P. Satturwar, M. N. Eddine, F. Ravenelle, J. C. Leroux, Eur. J. Pharm. Biopharm. 2007, 65, 379 .

[44] V. P. Sant, D. Smith, J. C. Leroux, J. Control. Release 2004, 97, 301.

[45] B. Liu, M. Yang, R. Li, Y. Ding, X. Qian, L. Yu, X. Jiang, Eur. J. Pharm. Biopharm. 2008, 69, 527.

[46] H. Wei, X. Z. Zhang, H. Cheng, W. Q. Chen, S. X. Cheng, R. X. Zhuo, J. Control. Release 2006, 116, 266.

[47] H. Wei, X. Z. Zhang, Y.Zhou, S. X. Cheng, R. X. Zhuo, Biomaterials 2006, 27, 2028.

[48] J. E. Chung, M. Yokoyama, M. Yamato, T. Aoyagi, Y. Sakurai, T. Okano, J. Control. Release 1999, 62, 115.

[49] N. Munshi, N. Rapoport, W. G. Pitt, Cancer Lett. 1997, 118, 13.

[50] Z. G. Gao, H. D. Fain, N. Rapoport, J. Control. Release 2005, 102, 203.

[51] H. Otsuka, Y. Nagasaki, K. Kataoka, $A d v$. Drug Deliv. Rev. 2003, 55, 403.

[52] D. Le Garrec, S. Gori, L. Luo, D. Lessard, D. C. Smith, M. A. Yessine, M. Ranger, J. C. Leroux, J. Control. Release 2004, 99, 83.

[53] T. W. Chung, K. Y. Cho, H. C. Lee, J. W. Nah, J. H. Yeo, T. Akaike, C. S. Cho, Polymer 2004, 45, 1591. 
[54] G. Zuccari, R. Carosio, A. Fini, P. G. Montaldo, I. Orienti, J. Control. Release 2005, 103, 369.

[55] Y. C. Wang, L. Y. Tang, T. M. Sun, C. H. Li, M. H. Xiong, J. Wang, Biomacromolecules 2008, 9, 388.

[56] Z. Hubalek, Cryobiology 2003, 46, 205.

[57] Y. S. Nam, H. S. Kang, J. Y. Park, T. G. Park, S. H. Han, I. S. Chang, Biomaterials 2003, 24, 2053.

[58] Y. Y. Li, X. Z. Zhang, G. C. Kim, H. Cheng, S. X. Cheng, R. X. Zhuo, Small 2006, 2, 917.

[59] S. Q. Liu, Y. W. Tong, Y. Y. Yang, Biomaterials 2005, 26, 5064

[60] W. D. Jang, Y. Nakagishi, N. Nishiyama, S. Kawauchi, Y. Morimoto, M. Kikuchi, K. Kataoka, J. Control. Release 2006, 113,73 .

[61] L. Zhang, T. L. U. Nguyen, J. Bernard, T. P. Davis, C. Barner-Kowollik, M. H. Stenzel, Biomacromolecules 2007, 8, 2890.

[62] T. Y. Jiang, Z. Y. Wang, C. Chen, F. K. Mo, Y. L. Xu, L. X. Tang, J. j. Liang, J. Appl. Polym. Sci. 2006, 101, 2878.

[63] S. H. Kim, J. H. Jeong, H. Mok, S. H. Lee, S. W. Kim, T. G. Park, Biotechnol. Prog. 2007, 23, 232.

[64] J. P. Santerre, K. Woodhouse, G. Laroche, R. S. Labow, Biomaterials 2005, 26, 7457.

[65] K. Lee, H. S. Choi, J. Y. Kim, I. S. Ahn, J. Hazard. Mater. 2003, 105, 179.

[66] F. Najafi, M. N. Sarbolouki, Biomaterials 2003, 24, 1175

[67] A. V. Kabanov, E. V. Batrakova, V. Y. Alakhov, J. Control. Release 2002, 82, 189.

[68] D. A. Chiappetta, A. Sosnik, Eur. J. Pharm. Biopharm. 2007, 66, 303.

[69] A. A. Exner, T. M. Krupka, K. Scherrer, J. M. Teets, J. Control. Release 2005, 106 , 188.

[70] A. Lavasanifar, J. Samuel, G. S. Kwon, Adv. Drug Deliv. Rev. 2002, 54, 169.

[71] H. Yin, E. S. Lee, D. Kim, K. H. Lee, K. T. Oh, Y. H. Bae, J. Control. Release 2008, 126, 130.
[72] Z. G. Gao, D. H. Lee, D. I. Kim, Y. H. Bae, J. Drug Target. 2005, 13, 391.

[73] M. D. Brown, A. Schatzlein, A. Brownlie, V. Jack, W. Wang, L. Tetley, A. I. Gray, I. F. Uchegbu, Bioconjugate Chem. 2000, 11,880 .

[74] P. Opanasopit, M. Yokoyama, M. Watanabe, K. Kawano, Y. Maitani, T. Okano, Pharm. Res. 2004, 21, 2001.

[75] S. R. Croy, G. S. Kwon, Curr. Pharm. Design 2006, 12, 4669.

[76] J. H. Park, S. Lee, J. H. Kim, K. Park, K. Kim, I. C. Kwon, Prog. Polym. Sci. 2008 , $33,113$.

[77] X. Li, K. Y. Mya, X. Ni, C. He, K. W. Leong, J. Li, J. Phys. Chem. B 2006, 110, 5920.

[78] C. Chen, C. H. Yu, Y. C. Cheng, P. H. F. Yu, M. K. Cheung, Biomaterials 2006, 27, 4804.

[79] W. J. Lin, C. L. Wang, L. W. Juang, J. Appl. Polym. Sci. 2006, 100, 1836.

[80] F. Zeng, H. Lee, M. Chidiac, C. Allen, Biomacromolecules 2005, 6, 2140.

[81] H. Lee, F. Zeng, M. Dunne, C. Allen, Biomacromolecules 2005, 6, 3119.

[82] B. H. Li, E. H. Moriyama, F. G. Li, M. T. Jarvi, C. Allen, B. C. Wilson, Photochem. Photobiol. 2007, 83, 1505.

[83] H. M. Aliabadi, A. Mahmud, A. D. Sharifabadi, A. Lavasanifar, J. Control. Release 2005, 104, 301.

[84] H. M. Aliabadi, S. Elhasi, A. Mahmud, R. Gulamhusein, P. Mahdipoor, A. Lavasanifar, Int. J. Pharm. 2007, 329 , 158.

[85] X. Shuai, H. Ai, N. Nasongkla, S. Kim, J. Gao, J. Control. Release 2004, 98 , 415.

[86] D. Sutton, S. H. Wang, N. Nasongkla, J. M. Gao, E. E. Dormidontova, Exp. Biol. Med. 2007, 232, 1090.

[87] Y. Geng, D. E. Discher, Polymer 2006, 47, 2519.

[88] K. Letchford, R. Liggins, H. Burt, $J$. Pharm. Sci. 2008, 97, 1179.

[89] Z. Ma, A. Haddadi, O. Molavi, A. Lavasanifar, R. Lai, J. Samuel, J. Biomed. Mater. Res. A 2007, 86, 300.
[90] J. S. Lee, J. W. Bae, Y. K. Joung, S. J. Lee, D. K. Han, K. D. Park, Int. J. Pharm. 2008, 346, 57.

[91] H. Lee, M. Hu, R. M. Reilly, C. Allen, Mol. Pharm. 2007, 4, 769.

[92] L. Chen, Z. Xie, J. Hu, X. Chen, X. Jing, J. Nanopart. Res. 2007, 9, 777.

[93] From the website of the US National Institutes of Health, http://clinicaltrials. gov with the keyword 'genexol', 2008

[94] P. Jie, S. S. Venkatraman, F. Min, B. Y. C. Freddy, G. L. Huat, J. Control. Release 2005, 110, 20 .

[95] E. S. Lee, K. T. Oh, D. Kim, Y. S. Youn, Y. H. Bae, J. Control. Release 2007, 123, 19.

[96] C. L. Lo, C. K. Huang, K. M. Lin, G. H. Hsiue, Biomaterials 2007, 28, 1225.

[97] E. Blanco, E. A. Bey, Y. Dong, B. D. Weinberg, D. M. Sutton, D. A. Boothman, J. Gao, J. Control. Release 2007, 122, 365.

[98] W. C. Lee, Y. C. Li, I. M. Chu, Macromol. Biosci. 2008, 6, 846.

[99] Z. L. Yang, X. R. Li, K. W. Yang, Y. Liu, J. Biomed. Mater. Res. 2008, in press, doi:10.1002/jbm.a.31504.

[100] G. Zhang, R. Zhang, X. Wen, L. Li, C. Li, Biomacromolecules 2008, 9, 36.

[101] J. H. Jeong, T. G. Park, J. Control. Release 2002, 82, 159

[102] E. S. Lee, H. J. Shin, K. Na, Y. H. Bae, J. Control. Release 2003, 90, 363.

[103] S. Q. Liu, Y. W. Tong, Y. Y. Yang, Molecular BioSystems 2005, $1,158$.

[104] H. S. Yoo, T. G. Park, J. Control. Release 2004, 96, 273.

[105] T. Trimaille, R. Gurny, M. Möller, J Polym. Sci. Part A: Polym. Chem. 2004, 42, 4379.

[106] T. Trimaille, K. Mondon, R. Gurny, M Möller, Int. J. Pharm. 2006, 319, 147. 